\title{
Single-row vs. double-row refixation of the subscapularis tendon after primary anatomic shoulder arthroplasty
}

\author{
Yacine Ameziane ${ }^{1} \cdot$ Kristian Nikolaus Schneider $^{1} \cdot$ Georg Gosheger ${ }^{1} \cdot$ Annika Mischke $^{2} \cdot$ Dominik Schorn $^{1}$. \\ Carolin Rickert ${ }^{1} \cdot$ Dennis Liem ${ }^{3}$
}

Received: 27 July 2019 / Published online: 28 April 2020

(c) The Author(s) 2020

\begin{abstract}
Background The postoperative integrity of the subscapularis tendon after primary anatomical shoulder arthroplasty has a significant effect on postoperative results. A transosseus Single Row Refixation technique (SRR) has shown up to $30 \%$ of partial tears in literature, a modified Double Row Refixation technique (DRR) has biomechanically shown a significantly reduced tear rate, but is yet to be proven in a clinical setting. Thus, we compared the SRR to the DRR technique using clinical outcome parameters and ultrasound examination.

Materials and methods 36 patients (40 shoulders; 20f, $16 \mathrm{~m}$; Øage: 66 years) were included in our retrospective cohort study. 20 shoulders were treated with the SRR technique (12f, $8 \mathrm{~m}$; FU ø40.9 months) and 20 with the DRR technique (11f, $9 \mathrm{~m}$; FU ø31.6 months). The SRR was performed with three to five transosseus mattress sutures. DRR consisted of two medial placed transosseus sutures and four laterally placed single tendon-to-tendon sutures. The postoperative subscapularis integrity was evaluated by ultrasound examination, the clinical outcome was assessed with the Constant-Murley Score (CS) and the American Shoulder and Elbow Surgeons Score (ASES).

Results The subscapularis tendon was intact in 14 patients (70\%) after SRR, whereas 18 patients (90\%) treated with the DRR demonstrated a sonographically intact postoperative subscapularis tendon. The CS was 61.4 points in the SRR cohort and 67.3 points in the DRR cohort $(p=0.314)$. No significant differences were found in both cohorts preoperative ( $\varnothing \mathrm{SRR}$ : 21.3 points; $\varnothing$ DRR: 16.2 points, $p=0.720$ ) and postoperative absolute ASES Scores ( $\varnothing$ SRR: 70.2 points; $\varnothing$ DRR: 73.0 points, $p=0.792)$. However, the DRR cohort showed a statistical tendency to a higher postoperative ASES increase than the SRR cohort ( $\varnothing$ SRR-ASES increase: 48.9 points; $\varnothing$ DRR-ASES increase: 56.8 points, $p=0.067$ ).

Conclusion The results of this study show that application of the DRR technique can significantly reduce the total rate of postoperative subscapularis tears what effects a clinical tendency towards higher ASES improvements and a better range of motion compared to the SRR technique.
\end{abstract}

Keywords Shoulder arthroplasty $\cdot$ Subscapularis $\cdot$ Shoulder $\cdot$ Ultrasound · Tenotomy $\cdot$ Refixation

\section{Introduction}

Yacine Ameziane

Yacine.ameziane@ukmuenster.de

1 Department of Orthopedics and Tumor Orthopedics, University Hospital Muenster, Albert-Schweitzer-Campus 1a, 48149 Muenster, Germany

2 Orthopaedic Practice Clinic, Muenster, Germany

3 Sporthopaedicum Berlin, Berlin, Germany
Shoulder arthroplasty has become a widely used and acknowledged procedure with good to excellent mid- to long-term results for patients with primary or secondary glenohumeral osteoarthritis $[20,22]$. The deltopectoral approach is commonly considered as the standard approach for shoulder arthroplasty including subscapularis take-down and refixation. The subscapularis is the most powerful muscle of the rotator cuff acting as an internal rotator, a depressor and a stabilizer of the glenohumeral joint and the refixation remains a key area of concern as insufficient refixation can negatively impact 
the postoperative outcome leading to anterior instability $[4,15,18]$. Several studies have already tested different surgical techniques of subscapularis refixation and reported postoperative signs of clinical subscapularis insufficiency in 9-67\% and at least partially compromised structural integrity in $13-47 \%$ of cases $[2,5,6,8,12-14$, $16,17]$.

Thus, the best surgical technique of subscapularis refixation still remains unclear: although the transosseus Single Row Refixation (SRR) constitutes a long established technique, recent literature has shown postoperative partial tears of the subscapularis tendon in up to $30 \%$ of all cases [13]. In an effort to improve structural integrity of the SRR, the Double Row Refixation (DRR) was biomechanically introduced by Ahmad et al. [1]. Their cadaveric study presents a significantly higher fixation strength of the DRR technique under cyclic loading compared to the simple transosseus SRR technique. However, literature lacks valuable clinical studies that transmit these biomechanical findings into a clinical setting. In our clinical setting, we changed our technique to the DRR technique in an effort to improve structural and possibly clinical results. We conducted this study to compare our earlier results with the SRR technique to the DRR technique regarding the postoperative integrity of the subscapularis tendon and the subsequent clinical outcome. The hypothesis of this study was that the DRR technique would result in a higher rate of intact subscapularis tendons after anatomic shoulder arthroplasty and provide at least equally good clinical results.

\section{Materials and methods}

Our study is based on two datasets-one 'historical' SRR cohort and a 'current' DRR cohort—comprising 20 shoulders each. All patients received primary hemiarthroplasty $(n=16)$ or total shoulder arthroplasty (TSA) $(n=24)$ in our clinic. The SRR cohort was surgically treated between 05.11.2001 and 09.02.2005 while DRR cohorts patients were operated between 02.10.2009 and 17.12.2014. The indication for surgery was either primary osteoarthritis $(n=34)$ or necrosis of the humeral head $(n=6)$. All patients were surgically treated by the same orthopedic consultant. Patients, who displayed posttraumatic arthritis, rheumatic arthritis or cuff tear arthropathy were excluded from this study as well as patients with a history of previous shoulder surgery.

The 'historical' SRR cohort consisted of 19 patients (20 shoulders; 10f, 9m), with an average age of 69.5 years $( \pm 8.41)$ and a follow-up of 41.0 months $( \pm 12.31) .12$ patients were operated on their dominant shoulder and eight on their non-dominant one. One patient received bilateral shoulder arthroplasty. In this group, subscapularis refixation was performed using the SRR technique: Three to five transosseus sutures (No. 2 Ethibond ${ }^{\circledR}$, Ethicon, Inc., Somerville, NJ, USA) were placed through the proximal humerus before implanting the prosthesis and re-attached to the tendon via mattress stitching technique (Fig. 1a; Table 1).

The 'current' DRR cohort consists of 17 patients (20 shoulders; $9 \mathrm{f}, 8 \mathrm{~m})$. Patients had an average age of 63 years $( \pm 10.26)$. The average follow-up was 31.6 months $( \pm 15.5)$. $\mathbf{a}$

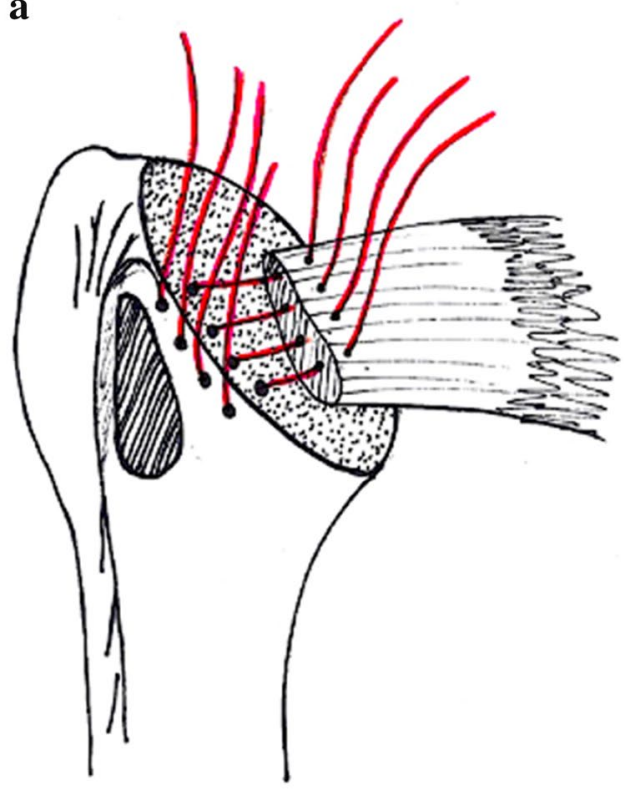

b

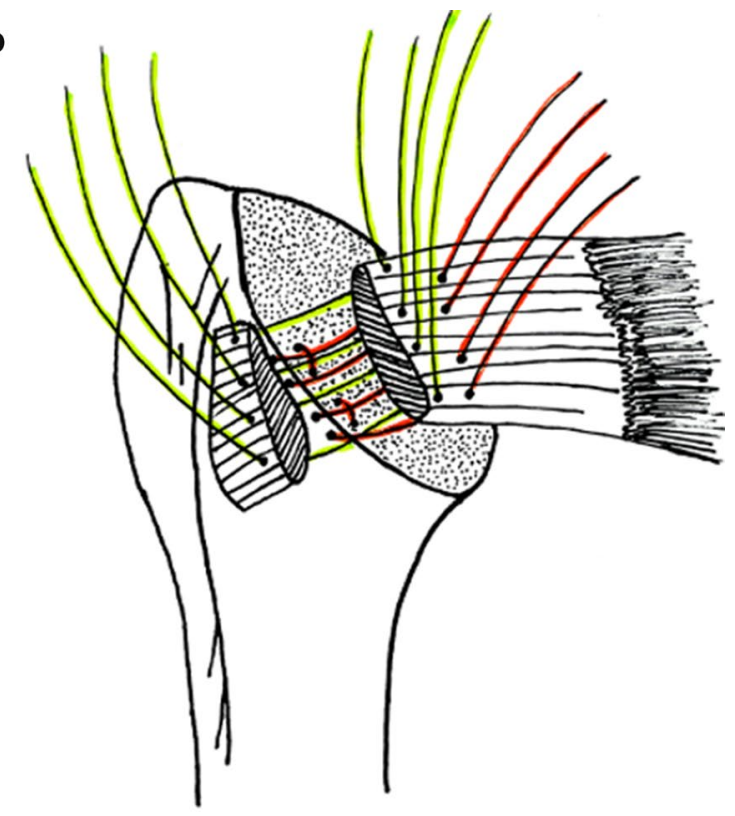

Fig. 1 a Single Row Refixation. b Double Row Refixation 
Table 1 Demographics

\begin{tabular}{llll}
\hline & SRR $( \pm)$ & DRR $( \pm)$ & $p$ \\
\hline Age (years) & $69.5(8.41)$ & $63(10.26)$ & 0.072 \\
FU (months) & $41(12.31)$ & $31.6(15.5)$ & 0.014 \\
F/M & $10 / 9$ & $9 / 8$ & \\
Dominant/non-domi- & $12 / 8$ & $13 / 7$ & \\
$\quad$ nant shoulder treated & & & \\
TSA & 12 & 12 & \\
HAS & 8 & 8 &
\end{tabular}

$F U$ follow-up, $f / m$ female/ male, TSA total shoulder arthroplasty, $H A S$ hemi shoulder arthroplasty

The dominant shoulder was affected in 13 cases, the nondominant one in seven cases. Three patients received bilateral shoulder arthroplasty. In this cohort, the subscapularis tendon was re-attached using a DRR technique with a medial and a lateral row: The medial row consisted of two transosseus sutures (No. 2 Ethibond $®$, Ethicon, Inc., Somerville, NJ, USA) and the additional lateral row consisted of two to four tendon-to-tendon sutures (Fig. 1b; Table 1).

\section{Operative technique}

All patients were operated in beach-chair position under general anesthesia. The deltopectoral approach was used to access the glenohumeral joint. The coracohumeral ligament was released while the coracoacromial ligament remained intact. After exposition of the subscapularis, two tagging sutures were placed through the medial part of the tendon, while other visible anatomic landmarks, such as the rotator interval, bicipital groove and circumflex vessels, were identified. The circumflex vessels were ligated and a tenotomy of the subscapularis tendon and capsule was performed medial to the lesser tuberosity. The tenotomy started next to the rotator cuff interval and ended next to the onset of M. latissimus dorsi, which was incised. In the SRR cohort the tenotomy was performed as a peel-off next to the insertion of the subscapularis with no tendon stump remaining on the lesser tuberosity. In the DRR cohort, the tenotomy was medialized about $5 \mathrm{~mm}$ to create a tendon stump for the placement of the lateral tendon-to-tendon sutures. After the rotator cuff interval was opened-up to the base of the coracoid, the glenohumeral ligaments were released from superior to inferior. The hypertrophic synovia and capsule were intraarticularly resected as needed to allow sufficient access to the glenoid.

While one type of prosthesis (Univers $\AA^{\circledR}$, Arthrex, Naples, FL, USA) was used in the SRR cohort, two different types of prosthesis (Global ap®, Depuy, Raynham, Massachussets, USA; Eclipse ${ }^{\circledR}$, Arthrex, Naples, Florida, USA) were implanted in the DRR cohort. 24 shoulders were treated with TSA, using a cementless glenoid interference screw in 12 cases and a cemented glenoid component in another 12 cases (pegged: 9; keeled: 3). 16 shoulders received hemiarthroplasty. After implanting the prosthesis, the subscapularis tendon was re-attached to the lesser tuberosity with the SRR and DRR technique, respectively.

Postoperative protocols were identical in both groups. The operated shoulder was immobilized in a sling for four weeks. Passive range of motion (ROM) was limited to $90^{\circ}$ flexion, $90^{\circ}$ abduction and $20^{\circ}$ of external rotation for the first six weeks after surgery. Subsequently, passive and active ROM were encouraged whereas heavy physical activity and sports were allowed after six months.

\section{Clinical assessment}

All patients underwent physical examination by one of two independent orthopedic surgeons. The obtained clinical results were evaluated through pre- and postoperative use of the American Shoulder and Elbow Surgeon Score (ASES). The Constant-Murley Score (CS) was postoperatively calculated for both shoulders.

All patients received postoperative radiographic examination with a true anterior posterior (true-a.p.) and Y view. The true-a.p. view was used to evaluate the acromio-humeral distance (AHD). An AHD of less than $5 \mathrm{~mm}$ was taken as a sign for rotator cuff tear arthropathy [10]. Axillary views were available in 15 cases of the SRR cohort and in 19 cases of the DRR cohort. According to a classification described by Habermeyer et al. all axillary views were analyzed for anterior dislocations as a potential sign for subscapularis insufficiency [9]. An anterior deviation of more than $5 \mathrm{~mm}$ to the glenoid center line was defined as an anterior decentralization of the humeral head [9].

All patients underwent an ultrasound examination of the operated and contralateral shoulder to assess the postoperative integrity of the subscapularis tendon (Fig. 2). Indicators of a fully torn tendon were absence and/or lack of mobility of the tendon during humeral rotation [11]. A partial tear was considered when less than $50 \%$ of the original subscapularis tendon thickness was present.

\section{Statistical analysis}

Statistical analysis was performed using SPSS 24.0 (SPSS Science, Chicago, IL, USA). The data were checked for normative variance with the Kolmogorow-Smirnow Test; and if normative variance was found, the $t$-test or the Mann-Whitney $U$ test were used for comparison and analysis. The level of significance was set at $p=0.05$. 


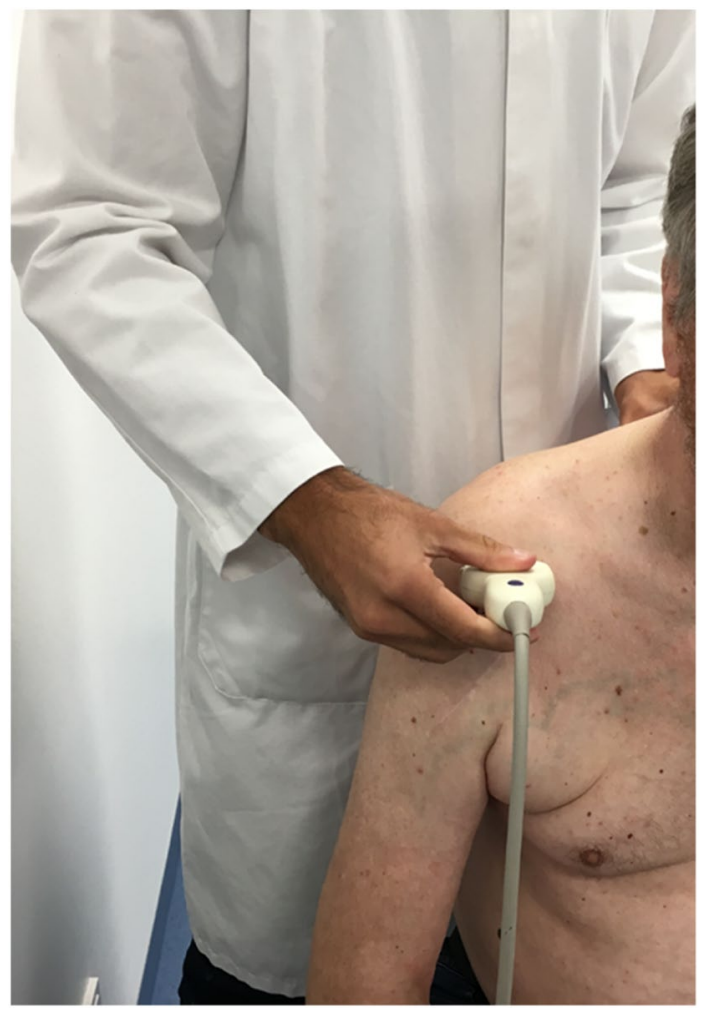

Fig. 2 Ultrasound examination

\section{Results}

\section{Subscapularis integrity}

Of the 20 shoulders in our SRR cohort, six shoulders (30\%) displayed sonographic signs of a partial subscapularis tear. 14 shoulders $(70 \%)$ showed adequate tendon thickness during ultrasound examination and passive movement of the tendon/muscle during physical examination.

Of the 20 shoulders in our DRR cohort, 18 shoulders (90\%) demonstrated an intact subscapularis tendon with complete integrity. One shoulder (5\%) was diagnosed with a partial tear. In one case (5\%) the subscapularis tendon was completely torn. No traumatic events that could have affected the subscapularis were reported in any of the aforementioned cases.

\section{Objective subscapularis function}

There was no significant difference $(p=0.314)$ between the postoperative CS of the SRR cohort (61.4 points) and the DRR cohort (67.3 points; Table 2).

However, comparison of the pre- to postoperative improvement in the ASES Scores of both cohorts showed a tendency towards statistical significance: The SRR cohort improved by an average of 39.3 points, whereas the DRR
Table 2 Objective clinical results

\begin{tabular}{llll}
\hline & SRR $( \pm)$ & DRR $( \pm)$ & $p$ \\
\hline Pain & $13.2(2.1)$ & $11.5(3.1)$ & 0.076 \\
ADL & $12.4(4.5)$ & $14.6(3.2)$ & 0.157 \\
ROM & $27.3(7.4)$ & $29.8(7.5)$ & 0.445 \\
Strength & $8.5(4.1)$ & $11.4(6.5)$ & 0.445 \\
Constant-Murley score (points) & $61.4(15.1)$ & $67.3(12.5)$ & 0.314 \\
Constant-Murley score (\%) & $72.4(17.3)$ & $78.5(14.3)$ & 0.461 \\
\hline
\end{tabular}

$A D L$ activities of daily living, $R O M$ range of motion

cohort increased by an average of 54.9 points $(p=0.067$; Table 3). These differences are further underlined by comparison of absolute ASES Scores: Pre-surgery, the average ASES Score of the SRR cohort (21.3 points) was better compared to the DRR cohort (16.2 points, $p=0.720$ ). Postoperatively, however, the average ASES Score of the DRR cohort (73.0 points) exceeded that of the SRR cohort (70.2 points, $p=0.792$ ).

\section{Range of motion}

Postoperatively, DRR patients displayed a significantly higher $(p=0.016)$ range of motion in abduction $\left(\varnothing 148^{\circ}\right)$ than SRR patients $\left(\varnothing 113^{\circ}\right)$. Correspondingly, DRR patient's flexion and external rotation exceeded that of SRR patients $\left(\varnothing 159^{\circ}\right.$ versus $\varnothing 138^{\circ}, p=0.176$ and $\varnothing 48^{\circ}$ versus $\varnothing 43^{\circ}$, $p=0.762)$. A tendency $(p=0.167)$ towards improved internal rotation was evident in the DRR cohort: While on average SRR patients were able to move their hand up to S1, the DRR cohort could, on average, reach up to Th12.

\section{Radiologic examination}

No significant differences could be found in the AHD. The average preoperative AHD was $9.4 \mathrm{~mm}$ (range $7.0-12.0 \mathrm{~mm}$ ) in the SRR cohort and $10.6 \mathrm{~mm}$ (range $7.0-15.8 \mathrm{~mm}$ ) in the DRR cohort. Postoperatively, no signs for a rotator cuff tear arthropathy were present in both cohorts: The SRR group displayed an average AHD of $9.30 \mathrm{~mm}$ (range 5.0-14 mm, $p=1$ ) the DRR cohort of $10.2 \mathrm{~mm}$ (range 5.6-16.7 mm, $p=0.191$ ). Regarding decentralization, seven patients (35\%) in the SRR cohort were anteriorly decentralized more than $5 \mathrm{~mm}$. Four of these seven patients (57\%) were diagnosed

Table 3 ASES score

\begin{tabular}{llll}
\hline & SRR $( \pm)$ & DRR $( \pm)$ & $p$ \\
\hline Preoperative & $21.3(17.8)$ & $16.2(11.7)$ & 0.720 \\
Postoperative & $70.2(19.8)$ & $73.0(14.1)$ & 0.792 \\
ASES increase & $39.3(22.7)$ & $54.9(18.0)$ & 0.067 \\
\hline
\end{tabular}


with a partial tear in ultrasound examination. In the DRR cohort, the axillary X-rays of four patients (20\%) displayed signs of anterior decentralization. While three of these patients had an intact tendon in ultrasound examination, one patient (20\%) had shown sonographic signs of a partial subscapularis tear.

\section{Discussion}

Due to its stabilizing and rotative effect the refixation of the M. subscapularis tendon crucially affects the postoperative outcome in shoulder arthroplasty [2]. The purpose of this study is the comparison of two different subscapularis refixation techniques: The SRR and the DRR technique. Subjective and objective parameters were obtained to assess the postoperative subscapularis tendon integrity and its impact on the clinical outcome.

The loss of function of the subscapularis is a well-known problem after shoulder arthroplasty. Miller et al. compared a large SRR cohort (32 patients) to nine patients who received a simple soft tissue tendon-to-tendon repair after subscapularis tenotomy [14]. Although the clinical results of the SRR treated patients exceeded those of soft tissue tendonto-tendon refixation, the SRR cohort still demonstrated positive clinical signs of subscapularis insufficiency in over $66 \%$ of all cases. They concluded that the subscapularis takedown is "a seldom recognized problem". In contrast, Caplan et al. published good to excellent clinical results of a soft tissue tendon-to-tendon refixation with negative postoperative signs for subscapularis insufficiency in $91-100 \%$ in 43 patients [6]. However, these results do not correspond with the findings of Miller et al. nor with similar studies like Jackson et al. who reported positive signs of a subscapularis tear in more than $40 \%$ of all patients treated with a soft tissue tendon-to-tendon refixation [12].

Armstrong et al. reviewed 30 shoulders regarding the subscapularis integrity after shoulder arthroplasty and a SRR with a mean age of 70.4 years and follow-up of 20.4 months [2]. Using ultrasound, an intact tendon was present in $87 \%$ of all cases and a full torn tendon in four patients (13\%). Our SRR cohort that is comparable aged (Armstrong et al.: $\varnothing 70.4$ years; et al.: $\varnothing 69.5$ years) and presents a longer followup (Armstrong et al.: $\varnothing 20.4$ months; et al.: $\varnothing 41.0$ months) showed improvable results with partial ruptures found in six cases (30\%). In contrast only two patients (10\%) of our DRR cohort (age: $\varnothing 63$ years; FU: $\varnothing 31.6$ months) demonstrated a partial or complete tear, postoperatively.

As a consequence of poor clinical results, the predominant SRR technique was challenged by many surgeons and evolved to a DRR technique that was first biomechanically tested by Ahmad et al. in a cadaveric study proving that the SRR technique has deficits regarding the strength of fixation.
In addition, the DRR technique achieves a higher grade of "tendon-to-humerus contact" after cyclic loading which let the authors presume a greater final strength after biological healing [1]. De Franco et al. established three criteria for ideal tendon healing: high initial fixation strength, minimal gap formation at the interface and stability until healing is completed [7]. All of those are achieved by the DRR technique.

Armstrong et al. published retrospective results of a DRR technique for subscapularis refixation after TSA in 30 shoulders [3]. The average ASES Score improved significantly from pre- to postoperatively from 45.3 to 76.8 points. Two complete ruptures $(7 \%)$ were found in 30 subscapularis tendons during ultrasound examination-similar results compared to our postoperative DRR tear rate of $10 \%$ (one complete rupture, one partial tear; preoperative $\varnothing$ ASES: 16.2 points to postoperative $\varnothing \mathrm{ASES}: 73.0$ points).

Evaluation of radiographic examination was also performed and correlated with structural integrity of the subscapularis. We could show moderate to low correlation between anterior decentralization and subscapularis integrity: While 57\% of SRR patients simultaneously displayed anterior decentralization and a partial subscapularis tear, only $20 \%$ of DRR patients showed such results. The degree of correlation between anterior decentralization and subscapularis integrity is further limited by the fact that, on a regular basis, decentralization of the humeral head is found preoperatively and cannot always be corrected postoperatively.

To our knowledge, this study is the first to confirm Ahmad et al.'s biomechanical findings in a clinical setting and to directly compare the tendon integrity following DRRbased surgeries versus SRR techniques. A further strength of this study is the high comparability of the two cohorts of patients - the only notable difference being the suture technique used for refixation. Finally, this study has some limitations. Firstly, the small number of patients and the inclusion of patients with osteoarthritis and humeral head necrosis as primary indications. Even though we would have wished the number of patients to be larger, the number of patients is comparable to other publications in this field. Literature is lacking studies that show postoperative differences between osteoarthritis and humeral head necrosis as indications for primary shoulder arthroplasty [21]. This study includes patients treated with hemi- or total shoulder arthroplasty. However, to our knowledge, no studies have yet been published indicating different subscapularis healing rates between hemi- or total shoulder arthroplasty. Findings across the two methods are thus considered comparable. Secondly, ultrasound-on which this study relied-provides only little information on muscle degeneration, which is very important for the subscapularis function. Ultrasound examination is nevertheless evaluated and described as a 
useful method of imaging the rotator cuff after shoulder arthroplasty [19]. Further, no specific tests for subscapularis strength and function were performed during clinical examination. This is in line with a previous study that presented low sensitivity, specificity and positive predictive value for clinical tests to assess the subscapularis integrity and function [2]. Thirdly, the ASES Score was the only surveyed parameter that allowed a pre- and postoperative comparison of the two cohorts. Thus, this study constitutes a platform for further research, e.g. for the use of more comparative methods as well as radiographic measures other than ultrasound to assess effects on muscle degeneration following different subscapularis refixation techniques.

\section{Conclusion}

This study clinically confirms that a modification of the intraoperative subscapularis refixation technique after anatomic shoulder arthroplasty from a SRR to a DRR technique can considerably reduce the total rate of postoperative subscapularis pathologies. We could sonographically confirm a reduction of incomplete healing from 30 to $10 \%$. Clinically we found a tendency towards higher ASES Score improvements and better range of motion in the DRR group.

\section{Acknowledgements Open Access funding provided by Projekt DEAL.}

\section{Compliance with ethical standards}

Conflict of interest D. Liem is a consultant for Conmed (Utica, New York, USA), Medacta (Castle San Pietro, Switzerland) and is part of the Editorial Board of Springer. Y. Ameziane, K. N. Schneider, G. Gosheger, A. Mischke, C. Rickert and D. Schorn declare that they have no conflict of interest.

Ethical approval All procedures performed in studies involving human participants were in accordance with the ethical standards of the institutional and/or national research committee and with the 1964 Helsinki declaration and its later amendments or comparable ethical standards.

Open Access This article is licensed under a Creative Commons Attribution 4.0 International License, which permits use, sharing, adaptation, distribution and reproduction in any medium or format, as long as you give appropriate credit to the original author(s) and the source, provide a link to the Creative Commons licence, and indicate if changes were made. The images or other third party material in this article are included in the article's Creative Commons licence, unless indicated otherwise in a credit line to the material. If material is not included in the article's Creative Commons licence and your intended use is not permitted by statutory regulation or exceeds the permitted use, you will need to obtain permission directly from the copyright holder. To view a copy of this licence, visit http://creativecommons.org/licenses/by/4.0/.

\section{References}

1. Ahmad CS, Wing D, Gardener TR, Levine WN, Bigliani LU (2007) Biomechanical evaluation of subscapularis repair used during shoulder arthroplasty. J Shoulder Elbow Surg 16:59-64. https://doi.org/10.1016/j.jse.2006.09.002

2. Armstrong A, Lashgari C, Teefey S, Menedez J, Yamaguchi K, Galatz LM (2006) Ultrasound evaluation and clinical correlation of subscapularis repair after total shoulder arthroplasty. J Shoulder Elbow Surg 15:541-548. https://doi.org/10.1016/j.jse.2005.09.013

3. Armstrong A, Southam JD, Horne AH, Hollenbeak CS, Flemming DJ, Kothari MJ (2016) Subscapularis function after total shoulder arthroplasty: electromyography, ultrasound, and clinical correlation. J Shoulder Elbow Surg 10:1674-1680. https://doi. org/10.1016/j.jse.2016.02.018

4. Brown TD, Bigliani LU (2000) Complications with humeral head replacement. Orthoped Clin North America 31:77-90

5. Buckley T, Miller R, Nicandri G, Lewis R, Voloshin I (2014) Analysis of subscapularis integrity and functionafter lesser tuberosity osteotomy versus subscapularis tenotomy in total shoulder arthroplasty using ultrsound and validated clinical outcome measures. J Shoulder Elbow Surg 23:1309-1317. https:// doi.org/10.1016/j.jse.2013.12.009

6. Caplan JL, Whitfield B, Neviaser RJ (2009) Subscapularis function after primary tendon to tendon repair in patients after replacement arthroplasty of the shoulder. J Shoulder Elbow Surg 18:193-197. https://doi.org/10.1016/j.jse.2008.10.019

7. DeFranco MJ, Higgins LD, Warner JJP (2010) Subscapularis Management in Open shoulder surgery. J Am Acad Orthop Surg 18:707-717

8. Gerber C, Yian EH, Pfirrmann CA, Zumstein MA, Werner CM (2005) Subscapularis muscle function after total shoulder replacement with lesser tuberosity osteotomy and repair. J Bone Joint Surg Am 87:1739-1745. https://doi.org/10.2106/ JBJS.D.02788

9. Habermeyer P, Magosch P, Lichtenberg S (2007) Recentering the humeral head for glenoid deficiency in total shoulder arthroplasty. Clin Orthop Relat Res 457:124-132. https://doi.org/10.1097/ BLO.0b013e31802ff03c

10. Hamada K, Fukuda H, Mikasa M, Kobayashi Y (1990) Roentgenographic findings in massive rotator cuff tears. A long-term observation. Clin Orthop Relat Res 254:92-96

11. Iannotti JP, Ciccine J, Buss DD, Visotsky JL, Mascha E, Cotman K, Rawool NM (2005) Accuracy of office-based ultrasonography of the shoulder for the diagnosis of rotator cuff tears. J Bone Joint Surg Am 87:1305-1311. https://doi.org/10.2106/JBJS.D.02100

12. Jackson JD, Cil A, Smith J, Steinmann SP (2010) Integrity and function of the subscapularis after total shoulder arthroplasty. J Shoulder Elbow Surg 19:1085-1090. https://doi.org/10.1016/j. jse.2010.04.001

13. Liem D, Kleeschulte K, Dedy N, Schulte TL, Steinbeck J, Marquardt B (2012) Subscapularis function in total shoulder arthroplasty: transosseus subscapularis repair in shoulder arthroplasty. J Shoulder Elbow Surg 21:1322-1327. https://doi.org/10.1016/j. jse.2011.09.022

14. Miller SL, Hazrati Y, Klepps S, Chiang A, Flatow EL (2003) Loss of subscapularis function after total shoulder replacement: a seldom recognized problem. J Soulder Elbow Surg 12:29-34. https://doi.org/10.1067/mse.2003.128195

15. Moeckle BH, AltcheckDW WRF, Wickiewicz TL, Dines DM (1993) Instabillity of the shoulder after arthroplasty. J Bone Joint Surgery Am 75:492-497 
16. Qureshi S, Hsaio A, Klug RA, Lee E, Braman J, Flatow EL (2008) Subscapularis function after total shoulder replacement: results with lesser tuberosity osteotomy. J Shoulder Elbow Surg 17:6872. https://doi.org/10.1016/j.jse.2007.04.018

17. Scalise JJ, Ciccone J, Iannotti JP (2010) Clinical, radiographic, and ultrasonographic comparison of subscapularis tenotomy and lesser tuberosity aosteotomy for total shoulder arthroplasty. J Bone Joint Surg Am 92:1627-1634. https://doi.org/10.2106/ JBJS.G.01461

18. Scheibel M, Habermeyer P (2008) Subscapularis dysfunction following anterior surgical approaches to the shoulder. J Shoulder Elbow Surg 17:671-683. https://doi.org/10.1016/j.jse.2007.11.005

19. Sofka MS, Adler RS (2003) Sonographic Evaluation of Shoulder Arthroplasty. Am J Roentgenol 180:1117-1120. https://doi. org/10.2214/ajr.180.4.1801117

20. Sowa B, Bochenek M, Bühlhoff M, Zeifang F, Loew M, Bruckner T, Raiss P (2017) The medium- and long term outcome of total shoulder arthroplasty for primary glenohumeral osteoarthritis in middle aged patients. Bone Joint J 99-B:939-943. https://doi. org/10.1302/0301-620X.99B7.BJJ-2016-1365.R1

21. Sowa B, Thierjung H (2017) Bülhoff, Loew M, Zeifang F, Bruckner T, Raiss P (2017) Functional results of hemi- and total shoulder arthroplasty according to diagnosis and patient age at surgery. Acta Orthop 88:310-314. https://doi.org/10.1080/17453 674.2017.1280656

22. Trochia ME, Cofield RH, Settergren CR (1997) Total shoulder arthroplasty with Neer prosthesis: long-term results. J Shoulder Elbow Surg 6:495-505

Publisher's Note Springer Nature remains neutral with regard to jurisdictional claims in published maps and institutional affiliations. 